\section{Round and about the heterocycles}

\section{Kevin T. Potts}

Heterocyclic Chemistry. By T.L. Gilchrist. Longman: 1985. Hbk £18.50, \$38.95; pbk $£ 8.50$.

OVER THE past decade increasingly sophisticated techniques have been brought to bear upon the synthesis of heterocyclic compounds, and upon the study of their properties and reactivity. Heterocyclic chemistry is no longer set apart from the main stream of organic chemistry, and organic chemists are finding many heterocyclic substrates to be important synthons. Dr Gilchrist, an active research worker in the field, has produced an excellent book. He builds on classical organic theory and provides a logical basis for understanding the varied reactions which heterocyclic systems undergo and the numerous strategies adopted for their synthesis.

Gilchrist has chosen his material well. $\mathrm{He}$ deals initially with the modern concepts of aromaticity and the criteria used to determine it, as well as with nonaromatic heterocycles where the consequences of bond angle strain in small- and large-ring heterocycles are clearly emphasized and torsional energy barriers are discussed for a variety of bonding situations.

In Chapter 4 the author develops a framework on which to build a rational approach to the vast array of synthetic procedures leading to heterocycles. The reactions involved in the ring-forming process are classified according to the synthetic method involved. Two broad groups of ring-forming reactions are considered: cyclizations, in which one bond is formed in the ring-closure process; and cycloadditions, in which two ring bonds are formed and in which no small molecules are eliminated. Although this scheme is an over-simplification well recognized by the author, it serves his purpose well in providing a systematic format to assist undergraduates and graduate students in assimilating a large amount of material.

Cyclization reactions are considered to involve intramolecular versions of the commonly encountered o-bond-forming processes, especially the reaction of an electrophilic atom with a nucleophilic one. This group includes bielectrophilic reagents in which the electrophilic centres are separated by one or more atoms, as well as binucleophiles such as hydroxylamines (a 1,2-binucleophile), and others with the nucleophilic centres separated by one or more atoms. Reagents with electrophilic and nucleophilic centres also fit into this category, as do the syntheses of benzo-fused heterocycles employing ana- logously substituted benzene derivatives. Used in illustration are examples of reactions involving displacements at saturated carbon, intramolecular nucleophilic addition to carbonyl groups and to other double bonds, cyclization onto triple bonds, radical cyclizations, carbene and nitrene cyclizations, and electrocyclic reactions. Numerous tables showing the reagents involved, possible intermediates and the products obtained complement and enlarge upon the discussion.

In keeping with the emphasis on cycloaddition reactions in the recent literature, Gilchrist focuses on their usefulness in leading to heterocycles with well-defined substitution patterns and, in many instances, with stereochemical control at more than one centre. Discussions of 1,3dipolar cycloadditions, hetero-DielsAlder reactions, [2+2] cycloadditions, cheliotropic reactions and the Ene reaction are well illustrated by numerous examples and by tables which provide a clear summary of the salient features.

The remaining chapters $(5-10)$ are a concise account of the synthesis and reactivity of all the commonly encountered three-, four-, five-, six- and sevenmembered heterocycles containing one or more heteroatoms, as well as their benzo-

\section{Behind mechanism}

\section{Neil S. Isaacs}

The Physical Basis of Organic Chemistry. By Howard Maskill. Oxford University Press:1985. Pp.490. Hbk £30, \$45; pbk $£ 14.95, \$ 24.95$.

Pictorial Orbital Theory. By John M. Tedder and Antony Nechvatal. Longman:1985. Pp.120. Hbk £14.95, $\$ 17.95 ; p b k £ 4.95$.

PHysical-organic chemistry is too large a subject to be encompassed in a single volume. Maskill's book aims to provide an account of the principles underlying techniques used routinely by physical-organic chemists in pursuit of reaction mechanisms. In this it fills a need, because many texts which discuss the evidence for accepted mechanisms cannot devote sufficient space to the theory on which the experimental evidence rests. The style and layout is pleasing, SI units are used throughout, and there are many worked examples (rather a lot in the gas-phase area) and tables of data. The author evidently expects a working knowledge of organic chemistry of at least second-year level in the reader.

The main strength of the book is to be found in the discussion of thermodynamic aspects of organic reactions and, although traditional in manner, the exposition is excellent. There is strong emphasis on fused derivatives; the summaries of the modern chemistry of this family of heterocycles will whet the reader's appetite to learn more. Finally, there is a brief but helpful introduction to heterocyclic nomenclature.

The book appears at a particularly opportune time in that it provides an introduction to the more detailed reference collection, Comprehensive Heterocyclic Chemistry, published last year*. Problems are provided at the end of each chapter, the index is quite comprehensive, and altogether this will prove an ideal text for undergraduates and beginning graduate students. The more seasoned research chemist will also find much of value here, particularly as there are numerous references to both the original literature and review articles.

Kevin T. Potts is a Professor in the Department of Chemistry, Rensselaer Polytechnic Institute, Troy, New York 12181, USA.

* For review see Nature 315, 354 (1985). Pergamon have published a companion volume to this work, Handbook of Heterocyclic Chemistry by Alan R. Katritzky, a 542-page paperback based on the general chapters in Com prehensive Heterocyclic Chemistry and designed to give an overall view of the subject for students and researchers. Price is $£ 22.95, \$ 29.95$.

partition functions, with which organic chemists would be well-advised to become familiar. Chemical potential, for example, emerges nicely and activity coefficients are clearly defined. Acid-base equilibria receive a fairly standard but clear treatment, though the hard-soft principle suffers from the lack of perturbation MO theory, the basis of any explanation of this phenomenon; it is inadequate to discuss "hardness" in terms of polarizability (itself not clearly defined). The theory of rates is adequately expounded, as is the basic thermodynamics of catalysis, and the Eigen and Marcus theories and the Brønsted Law are well presented. Isotope effects are derived from partition functions, but more could be made of their mechanistic use, in particular the secondary $\beta$-effect. LFER are discussed, though not multi-correlation analysis or the resonance parameter, $\sigma_{\mathrm{R}}$.

Regrettably, there are omissions. It has been the author's decision to exclude any discussion of molecular orbitals, to my mind a serious drawback, and there is little on the nature of intermolecular forces including solvation or the thermodynamic advantages of intramolecular processes. Conformational properties of ethanes are discussed; those of cyclohexanes but inadequately.

This is not, then, a new text on reaction mechanisms, nor does it encompass the entire "physical basis" of organic chemistry. Rather it will find a place as a helpful source of information on the basic physic- 